\title{
Route Optimization for Network Mobility Based Aeronautical Network Using Correspondent Router
}

\author{
Ruoshan Kong, Jing Feng, Huaibei Zhou \\ International School of Software, Wuhan University, Wuhan, China \\ E-mail:krs1024@public.wh.hb.cn \\ Received May 21, 2011; revised August 10, 2011; accepted September 26, 2011
}

\begin{abstract}
The future aeronautical network will be based on IPv6 and the services over the aeronautical network will be classified into 3 domains: Air Traffic Services (ATS), Airline Operational Services (AOS) and Passenger Information and Entertainment Services (PIES), among which the ATS and AOS domains are important for aircraft safety and airline business operation. Some schemes have been proposed to provide IP mobility support for aeronautical network, and Network Mobility (NEMO) scheme is the most promising one. However, using NEMO technology will lead to sub-optimal routing, so route optimization technology is highly desired for NEMO. A route optimization scheme is proposed for the ATS and AOS domains, which introduces the Correspondent Routers to realize the optimal routing and employs an improved procedure to reduce the handoff delay. The route optimization for the PIES domain is also discussed to provide better performance for some special scenarios.
\end{abstract}

Keywords: Aeronautical Network, Network Mobility, Route Optimization, Correspondent Router

\section{Introduction}

While the Internet has been widely used in more and more areas, civil aviation industry is still using traditional analogue technologies for communications, which will become a bottleneck for the future development of the industry. IPv6 will be an inevitable choice for the next generation of aeronautical network and has been discussed by the International Civil Aviation Organi- zation (ICAO) [1].

An important feature of the Aeronautical network is that the data flows to/from the aircraft are classified into 3 domains [2] (see Figure 1):

- Air Traffic Services Domain (ATS): this domain includes the data flows that are critical to the safety of the flight between the aircraft and the ground stations. The ground stations are deployed in different locations and an aircraft always connects to and communicates with the ground station that is geographically and topologically close to itself. Some ground stations have their own access networks and some stations make use of the access networks provided by ISPs, so generally speaking the ground stations are topologically close to their access networks.

- Airline Operational Services Domain (AOS): this do- main includes some data flows between an aircraft and the operational center of the airline corporation. These data flows are not critical to the safety but important for business and airline operations. Different from the ATS domain, the communication peer in the AOS domain may be geographically remote from the aircraft.

- Passenger Information and Entertainment Services domain (PIES): this domain is to provide Internet access to the passengers, so it is relatively less important, and the communication peers in this domain are globally located.

For safety and bandwidth consideration, an aircraft employs multiple link technologies for data communications, such as P34, LDL, WCDMA, WiMAX, and satellite, and the data flows of the 3 domains should be separately handled by different routing systems in an aircraft.

Several schemes have been proposed for the aeronauttical network to support IP communications, including the Border Gateway Protocol based, the Network Mobility (NEMO) based, and the Host Identity Protocol based. Among these schemes, the NEMO based scheme has advantages in many aspects such as mobility support, scalability and security, but the end-to-end packet delay is an important drawback of NEMO due to the 


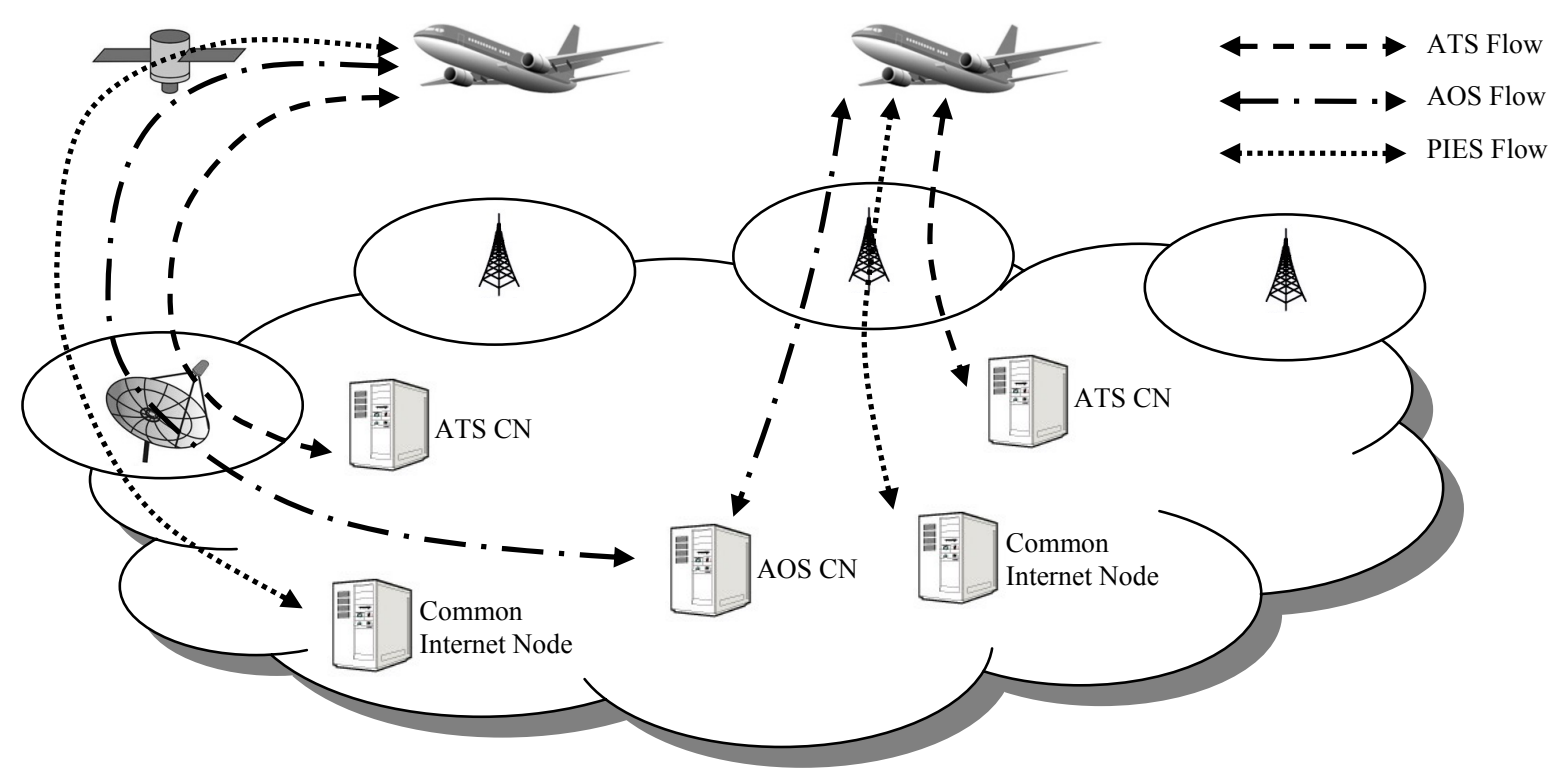

Figure 1. Topology of the aeronautical network.

sub-optimal routing [3]. This paper proposes a route optimization scheme for the NEMO based aeronautical network, which aims to set up optimal routing and reduce the handoff delay.

The rest of the paper is organized as follows: in Section 2 some related works are introduced, in Section 3 an optimization scheme for the ATS and AOS domains is introduced, in Section 4 some possible optimization methods for the PIES domain are discussed, and finally in Section 5 is the conclusion.

\section{Related Works}

\subsection{Network Mobility}

Network Mobility (NEMO) was proposed based on Mobile IPv6 [4] to support mobility for a Local Area Network (LAN). A Mobile Network consists of a Mobile Router (MR) and some Mobile Network Nodes that take the MR as the default router, through which the mobile network attaches to the Internet.

The home registration procedure is defined in the NEMO basic support protocol [5]: the MR gets a Care-of Address from the foreign network, and then sends a Binding Update (BU) message to its Home Agent (HA) to bind the Mobile Network's Prefix to the Care-of Address, then an IP-in-IP tunnel is set up between the MR and the HA, and all the traffic to/from the mobile network will be encapsulated into the tunnel and forwarded by the HA, which leads to the "triangular routing".

If the MR is equipped with multiple egress wireless interfaces, it can get multiple Care-of Addresses from different Access Networks. Following [6], the MR can make use of all these Care-of Addresses simultaneously and thus improve the availability and the bandwidth.

NEMO fits the aeronautical network quite well. The aircraft can be regarded as a Mobile Network consisting of a router and multiple LAN nodes. These LAN nodes can be sensor nodes in the ATS or AOS domain, or mobile devices taken by passengers in the PIES domain. The ground stations in the ATS domain, the operational center of the AOS domain and all the Internet hosts in the PIES domain can be regarded as the Correspondent Nodes $(\mathrm{CN})$.

\subsection{Typical Schemes to Provide IP Mobility Support}

[3] makes an overview on the typical schemes for aeronautical networks to support IPv6 mobility, including the Border Gateway protocol, the IPSec gateway scheme, the NEMO scheme, the SCTP protocol and the HIP protocol. This paper analyzes all these schemes on the following aspects:

- Session Continuity

- Mobile Network Support

- Multihoming

- Security

- End-to-end delay

- Scalability

- Applicability to PIES domain

- Convergence time 
- Efficiency

- Ground-initiated communications.

The authors conclude that the NEMO scheme has the best feasibility and overall performance, while the main drawback of this scheme is the end-to-end packet delay brought by the sub-optimal routing, so a route optimization scheme is desired.

\subsection{Route Optimization for NEMO Based Aeronautical Network}

Some route optimization schemes have been proposed, and the ORC scheme [7] is a typical one. This scheme introduces the Correspondent Router (CR) which covers a certain number of Correspondent Nodes (CN), and the MR can set up an IP-in-IP tunnel with the CR and bind the Mobile Network's prefix to the MR's Care-of Address so that the packets to/from the $\mathrm{CN}$ will be encapsulated into the MR-CR tunnel and forwarded by the CR without bypassing the HA (see Figure 2).

[8] employs the prefix delegation technology to expose the prefix of the access network to the Mobile Network Nodes, so that each Mobile Network Node can launch route optimization in the traditional Mobile IPv6 manner.

[9] proposes an optimization scheme similar to that in [8], but requests that the MR launch route optimization for each of the Mobile Network Nodes.

However, all these optimization schemes are proposed for general scenario and none of them are specially designed for the aeronautical networks, so [2] lists the required characteristics and desirable characteristics of route optimization for NEMO aeronautical networks.

[3] analyzes the above mentioned route optimization schemes and makes comparison on their applicability to aeronautical network. It concludes that [8] and [9] have signaling overhead problems because the route optimization has to be performed separately for each Mobile Network Node, while the ORC scheme has relatively good performance and some limitations in signaling security.

[3] also mentions another route optimization scheme for NEMO aeronautical networks-multi-HA scheme. Multiple HAs locate in different regions and the aircraft

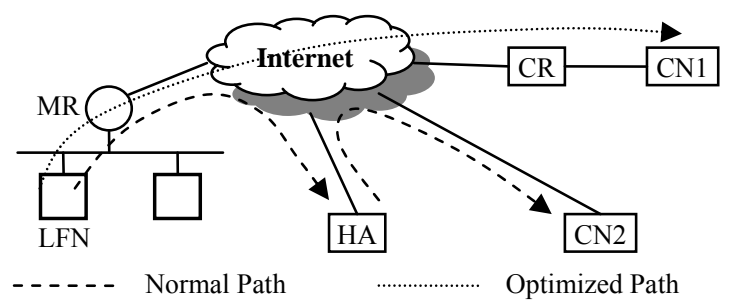

Figure 2. Routing of the ORC scheme.
(Mobile Network) switches between the HAs according to the geographical and topological position. This scheme cannot prevent the "triangular routing", but the aircraft can choose the nearest HA to prevent further deterioration on packet delay. This scheme looks nice but the feasibility is questionable because it's impossible for multiple HAs in different regions to cover the same Home Address, and the handoff between HAs will lead to the change of Home Address.

Some other route optimization schemes for NEMO aeronautical networks can also be found. [10] proposes to combine the NEMO and Ad hoc technologies, but this scheme only aims at the PIES domain and is suitable only for some special areas such as the North Atlantic.

\section{Route Optimization for ATS and AOS Domains}

Compared with the PIES domain, the ATS and AOS domains are different in the following aspects:

- ATS and AOS domains are much more important for safety and airline business and so require high availability, while the PIES domain is less important;

- ATS and AOS domains require relatively low bandwidth, while the PIES domain is bandwidth consuming;

- The CNs in the ATS and AOS domains are limited in number and are specially deployed by specific organizations, while the CNs in the PIES domain are common Internet nodes.

Considering the above differences, our scheme treats the PIES domain separately, and the optimization policies used by the PIES domain are different from what is employed in the ATS and AOS domains.

\subsection{Basic Mechanism for Route Optimization}

Our scheme requires each $\mathrm{CN}$ in the ATS or AOS domains is equipped with a Correspondent Router (CR), and the basic mechanism for route optimization is the same as that of the ORC scheme [7]. This requirement for infrastructure is reasonable and feasible because the $\mathrm{CNs}$ in the ATS and AOS domains are limited in number and deployed for special use.

The $\mathrm{CR}$ is the gateway router of the leaf network where the $\mathrm{CN}$ locates, so the $\mathrm{CR}$ can capture all the packets to/from the $\mathrm{CN}$.

An MR can send a Binding Update (BU) message to the $\mathrm{CR}$ which includes the Mobile Network Prefix and the Care-of Address. The CR replies with a Binding Acknowledgement (BA) message on receiving the BU, from which the MR can get the prefix of the leaf network managed by the CR. Hence the IP-in-IP tunnel 
between the MR and the CR is set up, and the MR will encapsulate all the packets from the Mobile Network Node to the CN with an outer IP header (source: MR's Care-of Address; destination: CR), while the CR will encapsulate all the packets from the $\mathrm{CN}$ to the Mobile Network Node with an outer IP header (source: CR; destination: MR's Care-of Address).

These encapsulated packets are sent to/from the MR's Care-of Address, so can be directly transferred without bypassing the HA, and the optimal routing is realized.

\subsection{CR Discovery and Return Routability Procedure}

A CR can be deployed by different organizations such as the government, the airline company, or the ISP, so it's quite probable that the aircraft doesn't know the CR's information and there must be some mechanism for the MR to find out the CR in front of the $\mathrm{CN}$. The ORC scheme [7] solves this issue by using the IPv6 anycast technology [11].

An anycast address-a predefined IPv6 suffix-is allocated to each $\mathrm{CR}$ besides its own unicast address. The MR can send an ICMP CR Discovery Request message to this anycast address (the CN's prefix + the predefined suffix), and the message will be captured and replied by the $\mathrm{CR}$, then the MR will get the $\mathrm{CR}$ information and launch route optimization to the $\mathrm{CR}$.

As we have mentioned in section 2.3, the signaling security is not fully discussed in [7]. In Mobile IPv6 [4], a mobile node must finish the "Return Routability Procedure" to prove it is the true owner of the Home Address and the Care-of Address before it can set up route optimization with a $\mathrm{CN}$. As Figure 3 shows, the mobile node sends a Home Test Init (HoTI) message with its Home Address and a Care-of Test Init (CoTI) message with its Care-of Address to the $\mathrm{CN}$, and the $\mathrm{CN}$ replies with Home Test (HoT) message and Care-of Test (CoT) message respectively. Both HoT and CoT contain a part of the authentication information, and the mobile node has to receive both messages to obtain the full authentication information needed in BU message.

The basic idea of the "Return Routability Procedure" can be used between the MR and the CR, but the details must be updated because it is insufficient for the MR to prove it owns both the Home Address and the Care-of Address. The MR must prove that it is the true router of the Mobile Network Prefix through this procedure.

[12] proposes an extension to the procedure, but [13] finds a potential risk in this extension and further improves it as follows (see Figure 4):

- The MR sends the HoTI message to the CR, which

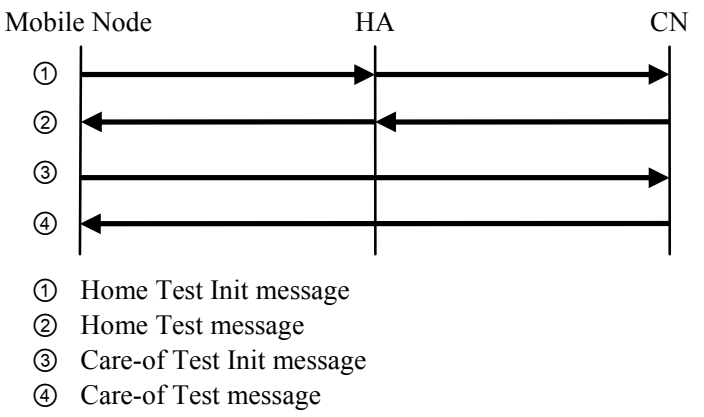

Figure 3. Return Routability Procedure signaling.

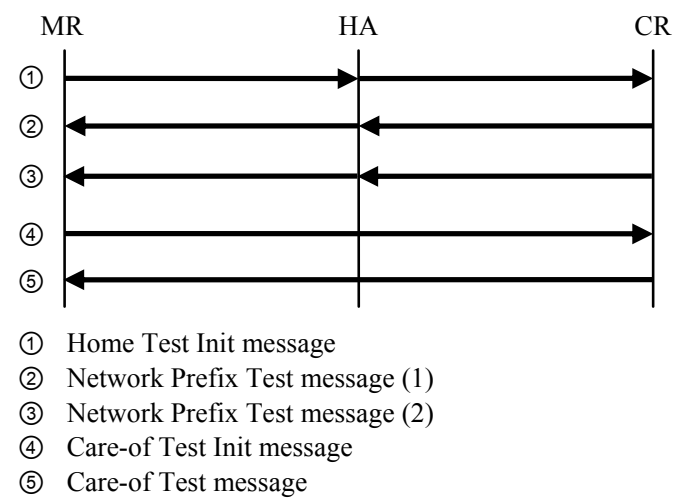

Figure 4. Signaling of improved Return Routability Procedure.

contains the Mobile Network Prefix and uses the MR's Home Address as the source address.

- The CR replies with two Network Prefix Test (NPT) messages on receiving the HoTI message, both of which contains a part of the authentication information. The two NPT messages are not sent to the MR's Home Address. The destination address of an NPT message is randomly generated based on the Mobile Network Prefix obtained from the HoTI message (i.e. Mobile Network Prefix + randomly generated suffix), and the highest bit of the randomly generated suffix of the 2 NPT messages must be 0 and 1 respectively.

- The MR sends the CoTI message to the CR, which uses the MR's Care-of Address as the source address.

- The CR replies with a CoT message which contains another part of the authentication information to the MR's Care-of Address.

The MR must receive the CoT message and capture the 2 NPT messages so as to collect all the authentication information, then it can send the BU message with full authentication information to the CR to set up optimal routing.

The security of the above procedure is proved in [13], and the signaling security limitation of the CR mechanism can be resolved with this improved "Return Rou- 
tability Procedure”.

\subsection{Handoff Acceleration}

When an aircraft switches from one Access Router to another, it has to perform the following steps:

- Step 1: Automatically configure the new Care-of Address from the new Access Router.

- Step 2: Wait for a period of time for the "Duplicate Address Detection (DAD)" [14].

- Step 3: Home registration.

- Step 4: Perform Return Routability Procedure with the CR.

- Step 5: Send BU message to the CR to restore the traffic to the $\mathrm{CN}$.

- Step 6: If the aircraft needs to switch to a new ground station $(\mathrm{CN})$, it should discover the new $\mathrm{CR}$ in front of the new CN.

- Step 7: Perform Return Routability Procedure with the new CR.

- Step 8: Send BU message to the new CR to set up optimal routing with the new $\mathrm{CN}$ (ground station).

So the total handoff delay can be expressed as:

$$
T=\left\{\begin{array}{r}
T_{\text {step } 1}+T_{\text {step } 2}+\cdots+T_{\text {step } 7}+T_{\text {step } 8} \\
(\text { switch to new } \mathrm{CN}) \\
T_{\text {step } 1}+T_{\text {step } 2}+\cdots+T_{\text {step } 5}+T_{\text {step } 8} \\
(\text { the same } \mathrm{CN})
\end{array}\right.
$$

The delay may lead to interrupt of communications with the ground station, which can bring potential risk, and also the aircraft has to suffer from sub-optimal routing to the new ground station from Step 3 to Step 8, which can affect the quality of communications.

In order to accelerate the handoff procedure, we raise some new requirements to the Access Network. Considering the Access Networks are specially deployed for future IPv6 based aeronautical network, we believe these requirements are acceptable.

- Every Access Router can inform the aircraft about the geographical scope it covers, by means of, for example, providing the coordinates of all the base stations and antennas, so that the aircraft can predict its handoff tendency with the help of GPS.

- Every Access Router maintains the basic information of all the neighboring Access Routers, including their IP addresses, geographical coverage and the prefixes managed by them.

- Every Access Router maintains a "Neighbor Aircraft List" which includes the IP addresses of the aircrafts that are currently served by a neighboring Access Router but may switch to this Access Router soon. Once an IP address is inserted into this list, it means the IP has been "booked" and the Access Router will protect the IP in the future "Duplicate Address Detection (DAD)" process.

The MR obtains from its Access Router and maintains the basic information of current and all the neighboring Access Routers, including their IP addresses, geographical coverage and the prefixes managed by them. The handoff process is modified as Figure 5 shows.

When an aircraft compares its coordinates with current Access Router $\left(\mathrm{AR}_{\mathrm{a}}\right)$ 's geographical coverage and finds that it will switch to another Access Router $\left(\mathrm{AR}_{\mathrm{b}}\right)$ soon, the $\mathrm{MR}$ calculates the automatically configured address $\left(\mathrm{IP}_{\mathrm{b}}\right)$ under $\mathrm{AR}_{\mathrm{b}}$ in advance and sends a "Neighbor DAD Request" message to $\mathrm{AR}_{\mathrm{a}}$ which contains $\mathrm{IP}_{\mathrm{b}}$ and $\mathrm{AR}_{\mathrm{b}}$ 's IP address. $\mathrm{AR}_{\mathrm{a}}$ forwards this message to $\mathrm{AR}_{\mathrm{b}}$. Then $\mathrm{AR}_{\mathrm{b}}$ performs the "Duplicate Address Detection (DAD)" for $\mathrm{IP}_{\mathrm{b}}$. If no duplicate address is found, $\mathrm{AR}_{\mathrm{b}}$ will reply with $\mathrm{a}$ "Neighbor DAD Complete" message to the MR and inserts $\mathrm{IP}_{\mathrm{b}}$ into the "Neighbor Aircraft List"; if a duplicate address is found, $\mathrm{AR}_{\mathrm{b}}$ will generate another available address $\left(\mathrm{IP}_{\mathrm{c}}\right)$ and send it to MR with a "Neighbor DAD Fail" message, also $\mathrm{IP}_{\mathrm{c}}$ will be inserted into the "Neighbor Aircraft List". In this way, the DAD process can be finished

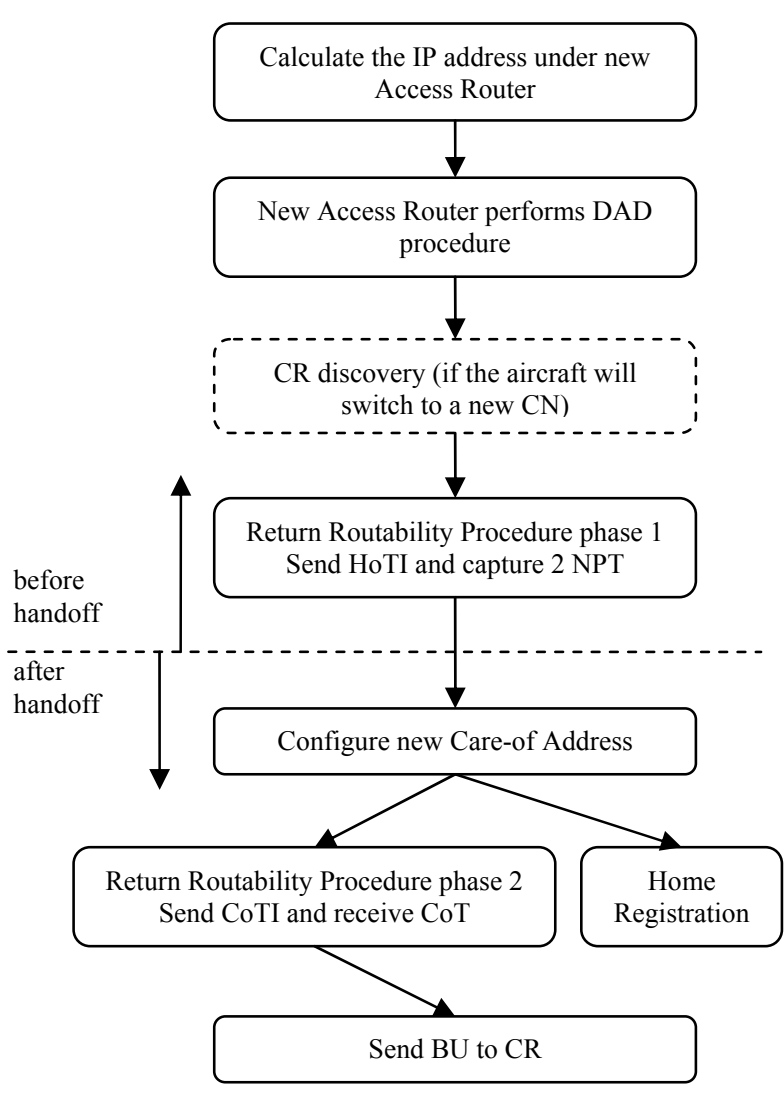

Figure 5. Optimized handoff process. 
before the handoff and the delay caused by DAD can be avoided.

If the aircraft finds that it will switch to a new $\mathrm{CN}$ (ground station), the MR can perform the CR discovery before handoff.

The MR has to perform Return Routability Procedure and update the binding for each CR after handoff, but the process can be optimized to shorten the delay. The MR can send out the HoTI message (with MR's Home Address as source address) and capture the 2 NPT messages before handoff, then when the MR switches to the new Access Router and finish configuring the new Care-of Address, it can send out the CoT message (to the $\mathrm{CR}$, with the Care-of Address as the source address) and the BU message (to the HA for Home Registration) simultaneously.

The whole handoff process is depicted in Figure 5 Compared with Equation (1), the handoff delay of the optimized process is:

$$
T=T_{\text {step } 1}+T_{\text {step } 3}+T_{\text {step } 8}
$$

\section{Route Optimization for PIES Domain}

The route optimization for the PIES domain is much harder because the $\mathrm{CNs}$ in this domain are globally located, and we cannot raise new requirement to the infrastructure.

Possible methods include multiple HAs scheme and CR based scheme, but generally speaking, these methods are effective only in some special scenarios.

\subsection{Multiple HAs Scheme}

As we have mentioned in Section 2.3, it's impossible for multiple HAs to cooperate for a single flow, and once the MR switches from one HA to another, it has to change the Home Address. However, in some special cases, we can let the MR register to multiple HAs simultaneously and let the passenger choose which HA to use.

For example, an aircraft is flying from China to France, and it has two HAs-one in China and one in Europe. The aircraft provides the passengers with two $\mathrm{Wi}-\mathrm{Fi}$ hotspots named "for Europe" and "for China", respectively. For a passenger who mainly access the websites in China, he should connect to the "for China" hotspot so as to get an IP address managed by the HA in China, and for a passenger who mainly access the websites in Europe, he should connect to the "for Europe" hotspot so as to get an IP address managed by the HA in Europe. In this way, the passenger can manually select the HA that is closer to the CNs, and thus optimize the routing to some extent.

\subsection{CR based Scheme}

Correspondent Routers can be deployed not only in the aeronautical networks, but also in common Internet. If a $\mathrm{CR}$ can be found, the route to/from the related CNs can be optimized. However, the load for CR discovery and route optimization can be very heavy considering the unlimited number of CNs.

So there must be some mechanism to limit the workload. For example, the MR can set an upper limit on the total amount of MR-CR tunnels, and the MR discovers CRs only for the sessions with the longest packet delay or largest throughput.

\section{Conclusions}

In this paper, we treat the route optimization problem for the ATS and AOS domains and for the PIES domain separately. For the ATS and AOS domains, we raise some new requirements to the Correspondent Routers and Access Routers, so that the optimal routing is realized while the handoff delay is reduced. Multihoming is supported by the HA, but the CR's support to multiple Care-of Addresses registration will be left for future research. For the PIES domain, the route optimization is harder because we cannot raise new requirement to the infrastructure, so only 2 possible methods for some special scenario are discussed.

\section{Acknowledgements}

This work was supported by a grant from the Major State Basic Research Development Program of China (973 Program) (No. 2009CB320400).

\section{References}

[1] International Civil Aviation Organization, "Manual for the ATN Using IPS Standards and Protocols (Doc 9896)," 1st Edition, Unedited Advance Version, February 2009.

[2] W. Eddy, W. Ivancic and T. Davis, "Network Mobility Route Optimization Requirements for Operational Use in Aeronautics and Space Exploration Mobile Networks," RFC 5522, October 2009.

[3] Christian Bauer and Martina Zitterbart, "A Survey of Protocols to Support IP Mobility in Aeronautical Communications," IEEE Communications Surveys \& Tutorials, Vol. PP, No. 99, 2010, pp. 1-16. doi:10.1109/SURV.2011.111510.00016

[4] D. Johnson, C. Perkins and J. Arkko, "Mobility Support in IPv6," RFC 3775, June 2004.

[5] V. Devarapalli, R. Wakikawa, A. Petrescu and P. Thubert, "Network Mobility (NEMO) Basic Support Protocol," RFC 3963, January 2005. 
[6] R. Wakikawa, V. Devarapalli, G. Tsirtsis, T. Ernst and K. Nagami, "Multiple Care-of Addresses Registration," RFC 5648, October 2009.

[7] R. Wakikawa, S. Koshiba, K. Uehara and J. Murai, "ORC Optimized Route Cache Management Protocol for Network Mobility," 10th International Conference on Telecommunications (ICT 2003), French Polynesia, Vol. 2, 23 February - 1 March 2003, pp. 1194-1200. doi:10.1109/ICTEL.2003.1191606

[8] K. Lee, J. Park and H. Kim, "Route Optimization for Mobile Nodes in Mobile Network Based on Prefix Delegation," IEEE 58th Vehicular Technology Conference, Orlando, 4-7 October 2003, pp. 2035-2038. doi:10.1109/VETECF.2003.1285382

[9] M. Calderon, C. J. Bernardos, M. Bagnulo, I. Soto and A. de la Oliva, "Design and Experimental Evaluation of a Route Optimization Solution for NEMO," IEEE Journal on Selected Areas in Communications, Vol. 24, No. 9, September 2006, pp. 1702-1716. doi:10.1109/JSAC.2006.875109
[10] F. Hoffmann, D. Medina and A. Wolisz, "Protocol Architecture Analysis for Internet Connectivity in Aeronautical Ad Hoc Networks," 2010 IEEE/AIAA 29th Digital Avionics Systems Conference (DASC), Salt Lake City, 3-7 October 2010, pp. (3.C.4-1)-(3.C.4-12). doi:10.1109/DASC.2010.5655370

[11] R. Hinden and S. Deering, "Internet Protocol Version 6 (IPv6) Addressing Architecture," RFC 3513, April 2003.

[12] C. Ng and J. Hirano, "Extending Return Routability Procedure for Network Prefix (RRNP)," Internet-Draft (work in progress) draft-ng-Nemo-rrnp-00, October

[13] R.04: Kong, J. Feng and H. B. Zhou, "A New Route Optimization Scheme for Network Mobility: Combining ORC Protocol with RRH Header Based on Quota Mechanism," Journal of Communications and Networks, Vol. 14, No. 1, 2012. (in press)

[14] S. Deering and R. Hinden, "Internet Protocol, Version 6 (IPv6) Specification,” RFC 2460, December 1998. 